\title{
Truth, Partial Logic and Infinitary proof systems
}

\author{
Martin Fischer \& Norbert Gratzl
}

August 24, 2017

\begin{abstract}
In this paper we apply proof theoretic methods used for classical systems in order to obtain upper bounds for systems in partial logic. We focus on a truth predicate interpreted in a Kripke style way via strong Kleene; whereas the aim is to connect harmoniously the partial version of KripkeFeferman with its intended semantics. The method we apply is based on infinitary proof systems containing an $\omega$-rule.
\end{abstract}

\section{Motivation}

This paper is part of a systematic investigation of syntactical predicates based on proof theoretic methods. In this paper we apply proof theoretic methods used for classical systems in order to obtain upper bounds for systems in partial logic. We focus on a truth predicate, interpreted in a Kripke style way via strong Kleene; whereas the aim is to connect harmoniously the partial version of Kripke-Feferman with its intended semantics. The method we apply is a variation of Schütte's based on infinitary proof systems.

The conception of truth we investigate is a version of Kripke's theory. Kripke made use of inductive definitions in order to characterize fixed-points as intended interpretations of the truth predicate. Of special interest for Kripke is the minimal fixed-point. We will use an infinitary proof system for which the set of theorems coincides with the minimal fixed-point. One part of our investigation designs an infinitary proof system, $\mathrm{SK}^{\infty}$. The system $\mathrm{SK}^{\infty}$ is a sequent calculus with an $\omega$-rule and truth introduction rules. With $\mathrm{SK}^{\infty}$ we can characterize the minimal strong Kleene fixed-point, $I_{s k}$. In a second part we consider an internal axiomatization of the semantical theory; the resulting thoery is called PKF. This theory is a good candidate and does not contain an infinitary rule, however it is not based on classical logic but on partial logic. The system PKF was introduced by Halbach \& Horsten [11] for achieving a faithful axiomatization of Kripke's fixed-point construction of Strong Kleene. The theory PKF has several interesting features for our purposes. On the one hand PKF is close to the semantic construction as it directly incorporates the closure conditions of the fixed-points. Moreover PKF is true in and only in the fixed-point models as an adequacy result shows. On the other hand PKF is close to the infinitary system $\mathrm{SK}^{\infty}$ as it can be embedded into it in a straight way. A closer look at 
the embedding allows for a more direct proof of a bound on the theorems of PKF, which is already known by the work of [11] and [5].

The plan of the paper is as follows:

In the preliminaries $(\S 2)$ we fix the notation and explain the basic concepts that we need in our presentation.

We develop in $\S 3$ the infinitary proof system $\mathrm{SK}^{\infty}$ for strong Kleene (allowing for cut elimination) and show the connection to the minimal fixed-point of strong Kleene.

We present a partial logic $(\S 4)$ in a sequent calculus style, PAL, that allows for cut elimination and is both sound and complete.

We then (in $\S 5$ ) formulate a sequent calculus of partial Kripke-Feferman (PKF) based on our calculus for partial logic.

The embedding of PKF into $\mathrm{SK}^{\infty}$ showing that every PKF-derivable sequent is also cut-free derivable in $\mathrm{SK}^{\infty}$ is carried out in $\S 6$.

Finally (in $\S 7$ ), we show for PKF theorems there is a bound on the height of proofs in $\mathrm{SK}^{\infty}$ showing that every theorem of PKF will be in a fixed-point construction $\alpha<\omega^{\omega}$, i.e. it will be appear in the fix point construction at a level below $\omega^{\omega}$.

\section{Preliminaries}

We use a language based on the signature with two two-place predicates $=$, $\neq$, two one-place predicates $\mathrm{T}, \mathrm{F}$ with the intended reading of truth and falsity. Moreover we have a finite number of function symbols used for coding purposes. Our languages contain the primitive logical symbols $\wedge, \vee, \forall, \exists$. Negation $\neg$ is an abbreviation in the following sense: $\neg \mathrm{T}(t):=\mathrm{F}(t) ; \neg \mathrm{F}(t):=\mathrm{T}(t) ; \neg s=t:=s \neq$ $t ; \neg s \neq t:=s=t$ and for complex sentences by DeMorgan rules and the usual interdefinability of quantifiers.

Let TERM $\mathrm{T}_{0}$ denote the set of all closed terms. Furthermore, SENT denotes the set of all sentences (of a given language); SENT denotes the complement of SENT. TRUE ${ }_{0}\left(\mathrm{FALSE}_{0}\right)$ is the set of true (false) atomic formulas in the language of arithmetic.

Coding and arithmetic: For an expression 'e' we use \#e for its Gödelnumber for some fixed standard Gödel-coding. For a natural number $n$ we use $\underline{n}$ for the $n$-th numeral. We assume that we have function symbols representing syntactical operations: and, or representing the p.r. functions that assign to the Gödel-numbers of two formulas the Gödel-number of their conjunction, disjunction. all, ex representing the p.r. functions that assign to the Gödel-number of a bound variable $x$ and the Gödel-number of a formula $A$ the Gödel-number of $\forall x A, \exists x A$. id, nid representing the p.r. functions that assign to the Gödelnumber of two closed terms $s, t$ the Gödel-number of $s=t, s \neq t$. $\operatorname{tr}, \mathrm{ntr}$ representing the p.r. functions that assign to the Gödel-number of a closed term 
$t$ the Gödel-number of $\mathbf{T}(t), \mathbf{F}(t)$. neg representing the p.r. function that assigns to the Gödel-number of a formula $A$ the Gödel-number of the formula $\neg A$ where $\neg A$ is short for the formula that represents the negation of $A$ in our language. val representing the p.r. function that assign to the Gödel-number of a closed term $t$ the number that $t$ denotes in the standard model. num representing the p.r. function that assigns to every number the Gödel-number of its numeral.

We also have sub representing the three-place substitution function that assigns to the Gödel-number of a term $s$ (or formula $A$ ), the Gödel-number of a free variable $v$ and the Gödel-number of a term $t$, the Gödel-number of the result of substituting all occurrences of $v$ in $s$ (or $A$ ) by $t$. We also write $s(\dot{x})$ $\operatorname{short}$ for $\operatorname{sub}(s, v, \operatorname{num}(x))$.

Moreover we have the formulas ct, sent, form representing the recursive sets of (Gödel-numbers of) closed terms, sentences and formulas. We also use form as an abbreviation for a formula with two free variables that represents the relation of being a formula with a designated free variable $v$.

\section{A sequent system for Strong Kleene}

In the following we introduce an infinitary sequent calculus, $\mathrm{SK}^{\infty}$. The system is a slight modification of the system that Cantini [6] introduced for the characterization of the minimal supervaluational fixed-point. We want to stress that in our infinitary language all formulas are closed sentences. Our sequent calculus is based on a Tait-language but in contrast to Cantini's system we use a two-sided calculus. ${ }^{1}$ Sequents are (finite) sets of formulas.

\section{$3.1 \mathrm{SK}^{\infty}$}

We structure the presentation of $\mathrm{SK}^{\infty}$ in the following way: initial sequents, followed by rules: structural, propositional, quantificational, truth followed by Cut.

\section{Initial Sequents}

$\begin{array}{ccc}(\mathrm{LAx}) & \Gamma, A \Rightarrow A, \Delta & A \text { atomic. } \\ \text { (Ax.Ar.1) } & \Gamma \Rightarrow A, \Delta & \text { if: } \# A \in \mathrm{TRUE}_{0} \\ \text { (Ax.Ar.2) } & \Gamma, A \Rightarrow \Delta & \text { if: } \# A \in \mathrm{FALSE}_{0} \\ \text { (SENT.1) } & \Gamma \Rightarrow \mathrm{F}(t), \Delta & \text { if: } t^{\mathcal{N}} \notin \mathrm{SENT} \\ \text { (SENT.2) } & \Gamma, \mathrm{T}(t) \Rightarrow \Delta & \text { if: } t^{\mathcal{N}} \notin \mathrm{SENT} \\ \text { (TID) } & \Gamma, \mathrm{T}(s) \Rightarrow \mathrm{T}(t), \Delta & \text { if: } s^{\mathcal{N}}=t^{\mathcal{N}} . \\ \text { (FID) } & \Gamma, \mathrm{F}(s) \Rightarrow \mathrm{F}(t), \Delta & \text { if: } s^{\mathcal{N}}=t^{\mathcal{N}} .\end{array}$

\section{Propositional}

\footnotetext{
${ }^{1}$ In his book Cantini uses a two-sided calculus, which is in this respect closer to our system.
} 


$$
\begin{array}{cc}
\frac{A, B, \Gamma \Rightarrow \Delta}{A \wedge B, \Gamma \Rightarrow \Delta}(\mathrm{L} \wedge) & \frac{\Gamma \Rightarrow \Delta, A \quad \Gamma \Rightarrow \Delta, B}{\Gamma \Rightarrow \Delta, A \wedge B}(\mathrm{R} \wedge) \\
\frac{A, \Gamma \Rightarrow \Delta \quad B, \Gamma \Rightarrow \Delta}{A \vee B, \Gamma \Rightarrow \Delta}(\mathrm{L} \vee) & \frac{\Gamma \Rightarrow \Delta, A, B}{\Gamma \Rightarrow \Delta, A \vee B}(\mathrm{R} \vee)
\end{array}
$$

\section{Quantificational}

$$
\begin{aligned}
& \frac{\Gamma, A_{x}(t) \Rightarrow \Delta}{\Gamma, \forall x A \Rightarrow \Delta}\left(\forall \mathrm{L}, t \in \mathrm{TERM}_{0}\right) \quad \frac{\ldots \Gamma \Rightarrow \Delta, A_{x}(t) \ldots}{\Gamma \Rightarrow \Delta, \forall x A}\left(\forall^{\omega} \mathrm{R}\right), t \in \mathrm{TERM}_{0} \\
& \frac{\Gamma \Rightarrow \Delta, A_{x}(t)}{\Gamma \Rightarrow \Delta, \exists x A}\left(\exists \mathrm{R}, t \in \mathrm{TERM}_{0}\right) \quad \frac{\ldots \Gamma, A_{x}(t) \Rightarrow \Delta \ldots}{\Gamma, \exists x A \Rightarrow \Delta}\left(\exists^{\omega} \mathrm{L}\right), t \in \mathrm{TERM}_{0}
\end{aligned}
$$

\section{Truth}

$$
\begin{array}{ll}
\frac{A \Rightarrow \Delta}{\Gamma, \mathrm{T}(t) \Rightarrow \Delta}(\mathrm{TL}), \text { if: } t^{\mathcal{N}}=\# A & \frac{\Gamma \Rightarrow A}{\Gamma \Rightarrow \mathrm{T}(t), \Delta}(\mathrm{TR}), \text { if: } t^{\mathcal{N}}=\# A \\
\frac{\neg A \Rightarrow \Delta}{\Gamma, \mathrm{F}(t) \Rightarrow \Delta}(\mathrm{FL}), \text { if: } t^{\mathcal{N}}=\# A & \frac{\Gamma \Rightarrow \neg A}{\Gamma \Rightarrow \mathrm{F}(t), \Delta} \text { (FR), if: } t^{\mathcal{N}}=\# A
\end{array}
$$

\section{Cut}

$$
\frac{\Gamma \Rightarrow \Delta, A \quad A, \Gamma \Rightarrow \Delta}{\Gamma \Rightarrow \Delta}(\mathrm{Cut})
$$

\subsection{Some results on $\mathrm{SK}^{\infty}$}

We state some standard definitions:

The rank of a formula $C, \operatorname{rk}(C)$ is defined as usual: $\operatorname{rk}(C)=0$ if $C$ is atomic and if $C$ is $A \wedge B$ or $A \vee B$ then $\operatorname{rk}(C)=\max \{\operatorname{rk}(A), \operatorname{rk}(B)\}+1$ and if $C$ is $\forall x A$ or $\exists x A$, then $\mathrm{rk}(C)=\mathrm{rk}(A)+1$.

The cut-rank of a derivation $d$ is defined as:

$$
\operatorname{crk}(d):=\sup \{\operatorname{rk}(C)+1 \mid C \text { a cut formula of } d\} .
$$

We restrict our attention to derivations with finite cut-rank.

The height of a derivation $d$ is defined recursively as:

$$
\operatorname{hgt}(d):=\sup \left\{h g t\left(d_{i}\right)+1\right\}
$$

such that the $d_{i}$ are the immediate subderivations of $d$. 
We also keep track of the number of applications of truth rules by the truth rank of a formula occurrence of a derivation. ${ }^{2}$

Before we begin with some standard proof theoretic results we give an inductive definition of $\vdash_{\rho, r}^{\alpha} \Gamma \Rightarrow \Delta$ is short for there is a derivation $d$ of $\Gamma \Rightarrow \Delta$ of $\operatorname{hgt}(d) \leq \alpha$ and $\operatorname{crk}(d) \leq r$ and truth $\operatorname{rank} \operatorname{tr}(d) \leq \rho$.

1. Axioms: For all $\alpha$ and $\rho, r<\omega$ :

$$
\begin{aligned}
& \mathrm{SK}^{\infty} \vdash_{\rho, r}^{\alpha} \Gamma, A \Rightarrow A, \Delta \text { if } A \text { is atomic, } \\
& \mathrm{SK}^{\infty} \vdash_{\rho, r}^{\alpha} \Gamma \Rightarrow A, \Delta \text { if: } \# A \in \mathrm{TRUE}_{0}, \\
& \mathrm{SK}^{\infty} \vdash_{\rho, r}^{\alpha} \Gamma, A \Rightarrow \Delta \text { if: } \# A \in \mathrm{FALSE}_{0}, \\
& \mathrm{SK}^{\infty} \vdash_{\rho, r}^{\alpha} \Gamma \Rightarrow \mathrm{F}(t), \Delta \text { if: } t^{\mathcal{N}} \notin \mathrm{SENT}, \\
& \mathrm{SK}^{\infty} \vdash_{\rho, r}^{\alpha} \Gamma, \mathrm{T}(t) \Rightarrow \Delta \text { if: } t^{\mathcal{N}} \notin \mathrm{SENT}, \\
& \mathrm{SK}^{\infty} \vdash_{\rho, r}^{\alpha} \Gamma, \mathrm{T}(s) \Rightarrow \mathrm{T}(t), \Delta \text { if: } s^{\mathcal{N}}=t^{\mathcal{N}}, \\
& \mathrm{SK}^{\infty} \vdash_{\rho, r}^{\alpha} \Gamma, \mathrm{F}(s) \Rightarrow \mathrm{F}(t), \Delta \text { if: } s^{\mathcal{N}}=t^{\mathcal{N}},
\end{aligned}
$$

2. Logical rules: For all $\alpha<\beta$ and $\rho, r<\omega$ :

$$
\begin{aligned}
& \text { if } \mathrm{SK}^{\infty} \vdash_{\rho, r}^{\alpha} \Gamma, A, B \Rightarrow \Delta \text { then } \mathrm{SK}^{\infty} \vdash_{\rho, r}^{\beta} \Gamma, A \wedge B \Rightarrow \Delta, \\
& \text { if } \mathrm{SK}^{\infty} \vdash_{\rho, r}^{\alpha} \Gamma \Rightarrow A, \Delta \text { and } \mathrm{SK}^{\infty} \vdash_{\rho, r}^{\alpha} \Gamma \Rightarrow B, \Delta, \\
& \text { then } \mathrm{SK}^{\infty} \vdash_{\rho, r}^{\beta} \Gamma \Rightarrow A \wedge B, \Delta, \\
& \text { if } \mathrm{SK}^{\infty} \vdash_{\rho, r}^{\alpha} \Gamma, A \Rightarrow \Delta \text { and } \mathrm{SK}^{\infty} \vdash_{\rho, r}^{\alpha} \Gamma, B \Rightarrow \Delta, \\
& \text { then } \mathrm{SK}^{\infty} \vdash_{\rho, r}^{\beta} \Gamma, A \vee B \Rightarrow \Delta, \\
& \text { if } \mathrm{SK}^{\infty} \vdash_{\rho, r}^{\alpha} \Gamma \Rightarrow \Delta, A, B \text { then } \mathrm{SK}^{\infty} \vdash_{\rho, r}^{\beta} \Gamma \Rightarrow \Delta, A \vee B, \\
& \text { if } \mathrm{SK}^{\infty} \vdash_{\rho, r}^{\alpha} \Gamma, A_{x}(t) \Rightarrow \Delta \text { then } \mathrm{SK}^{\infty} \vdash_{\rho, r}^{\beta} \Gamma, \forall x A \Rightarrow \Delta, \\
& \text { if } \mathrm{SK}^{\infty} \vdash_{\rho, r}^{\alpha} \Gamma \Rightarrow A_{x}(t), \Delta \text { for all } t \in \mathrm{TERM}_{0}, \\
& \text { then } \mathrm{SK}^{\infty} \vdash_{\rho, r}^{\beta} \Gamma \Rightarrow \forall x A, \Delta, \\
& \text { if } \mathrm{SK}^{\infty} \vdash_{\rho, r}^{\alpha} \Gamma, A_{x}(t) \Rightarrow \Delta \text { for all } t \in \mathrm{TERM}_{0}, \\
& \text { then } \mathrm{SK}^{\infty} \vdash_{\rho, r}^{\beta} \Gamma, \exists x A \Rightarrow \Delta, \\
& \text { if } \mathrm{SK}^{\infty} \vdash_{\rho, r}^{\alpha} \Gamma \Rightarrow A_{x}(t), \Delta \text { then } \mathrm{SK}^{\infty} \vdash_{\rho, r}^{\beta} \Gamma \Rightarrow \Delta, \exists x A
\end{aligned}
$$

3. Truth rules and cut:

\footnotetext{
${ }^{2}$ This is possible because in the truth rules we do not allow for contexts in the upper sequents. The reason for this is that if the principal formula of an application of a truth rule would occur already in the context of the upper sequent then the truth rank $\rho$ would not be uniquely determined.
} 
For all $\alpha<\beta, \rho<\kappa$ and $r<\omega$ :

(i) $\quad$ if $\mathrm{SK}^{\infty} \vdash_{\rho, r}^{\alpha} A \Rightarrow \Delta$ and $t^{\mathcal{N}}=\# A$, then $\mathrm{SK}^{\infty} \vdash_{\kappa, r}^{\beta} \Gamma, \mathrm{T}(t) \Rightarrow \Delta$,

(ii) if $\mathrm{SK}^{\infty} \vdash_{\rho, r}^{\alpha} \Gamma \Rightarrow A$ and $t^{\mathcal{N}}=\# A$, then $\mathrm{SK}^{\infty} \vdash_{\kappa, r}^{\beta} \Gamma \Rightarrow \mathrm{T}(t), \Delta$,

(iii) if $\mathrm{SK}^{\infty} \vdash_{\rho, r}^{\alpha} \neg A \Rightarrow \Delta$ and $t^{\mathcal{N}}=\# A$, then $\mathrm{SK}^{\infty} \vdash_{\kappa, r}^{\beta} \Gamma, \mathrm{F}(t) \Rightarrow \Delta$,

(iv) if $\mathrm{SK}^{\infty} \vdash_{\rho, r}^{\alpha} \Gamma \Rightarrow \neg A$ and $t^{\mathcal{N}}=\# A$, then $\mathrm{SK}^{\infty} \vdash_{\kappa, r}^{\beta} \Gamma \Rightarrow \mathrm{F}(t), \Delta$,

(v) $\quad$ if $\mathrm{SK}^{\infty} \vdash_{\rho, r}^{\alpha} \Gamma \Rightarrow A, \Delta$ and $\mathrm{SK}^{\infty} \vdash_{\rho, r}^{\alpha} \Gamma, A \Rightarrow \Delta$ and $\mathrm{rk}(A)<r$, then $\mathrm{SK}^{\infty} \vdash_{\rho, r}^{\beta} \Gamma \Rightarrow \Delta$,

The following observations are direct consequences of the definitions and $\mathrm{SK}^{\infty}$. The notation $\neg \Phi$ is short for $\{\neg A \mid A \in \Phi\}$.

\section{Lemma 1.}

(i) $\mathrm{SK}^{\infty} \vdash_{\rho, r}^{k} A_{x}(s) \Rightarrow A_{x}(t)$, if $s^{\mathcal{N}}=t^{\mathcal{N}}$, for any $A$ and for some $k, \rho, r<\omega$.

(ii) If $\mathrm{SK}^{\infty} \vdash_{\rho, r}^{\alpha} \Gamma \Rightarrow \Delta$, then $\mathrm{SK}^{\infty} \vdash_{\rho, r}^{\alpha} \neg \Delta \Rightarrow \neg \Gamma$. (Contraposition)

(iii) If $\mathrm{SK}^{\infty} \vdash_{\rho, r}^{\alpha} \Gamma \Rightarrow \Delta$, and $\alpha \leq \beta, \rho \leq \sigma r \leq l$, then $\mathrm{SK}^{\infty} \vdash_{\sigma, l}^{\beta} \Gamma \Rightarrow \Delta$ (Monotonicity).

(iv) If $\mathrm{SK}^{\infty} \vdash_{\rho, r}^{\alpha} \Gamma \Rightarrow \Delta$ and $\Gamma \subseteq \Gamma^{\prime}, \Delta \subseteq \Delta^{\prime}$, then $\mathrm{SK}^{\infty} \vdash_{\rho, r}^{\alpha} \Gamma^{\prime} \Rightarrow \Delta^{\prime}$. (Weakening)

(v) $\nvdash_{\rho, 0}^{\alpha} \Rightarrow$ (Consistency).

\subsection{Cut elimination}

In this subsection we show that for our infinitary calculus we get full cutelimination, not only partial. ${ }^{3}$

We start with a lemma establishing the axiomatic cases.

\section{Lemma 2.}

(i) If $\vdash_{\rho, r}^{\alpha} \Gamma \Rightarrow \Delta, A$ and $\# A \in \mathrm{FALSE}_{0}$, then $\vdash_{\rho, r}^{\alpha} \Gamma \Rightarrow \Delta$;

(ii) If $\vdash_{\rho, r}^{\alpha} A, \Gamma \Rightarrow \Delta$ and $\# A \in \operatorname{TRUE}_{0}$, then $\vdash_{\rho, r}^{\alpha} \Gamma \Rightarrow \Delta$;

(iii) If $\vdash_{\rho, r}^{\alpha} \mathrm{F}(t), \Gamma \Rightarrow \Delta$ and $t^{\mathcal{N}} \notin \mathrm{SENT}$, then $\vdash_{\rho, r}^{\alpha} \Gamma \Rightarrow \Delta$;

(iv) If $\vdash_{\rho, r}^{\alpha} \Gamma \Rightarrow \Delta, \mathrm{T}(t)$ and $t^{\mathcal{N}} \notin \mathrm{SENT}$, then $\vdash_{\rho, r}^{\alpha} \Gamma \Rightarrow \Delta$;

(v) If $\vdash_{\rho, r}^{\alpha} \Gamma \Rightarrow \Delta, \mathrm{T}(s), \mathrm{T}(t)$ and $s^{\mathcal{N}}=t^{\mathcal{N}}$, then $\vdash_{\rho, r}^{\alpha} \Gamma \Rightarrow \Delta, \mathrm{T}(s)$;

(vi) If $\vdash_{\rho, r}^{\alpha} \Gamma, \mathrm{T}(s), \mathrm{T}(t) \Rightarrow \Delta$ and $s^{\mathcal{N}}=t^{\mathcal{N}}$, then $\vdash_{\rho, r}^{\alpha} \Gamma, \mathrm{T}(s) \Rightarrow \Delta$;

\footnotetext{
${ }^{3}$ The presentation in this subsection is therefore closer to Cantini [6] than Cantini [5].
} 
(vii) If $\vdash_{\rho, r}^{\alpha} \Gamma \Rightarrow \Delta, \mathrm{F}(s), \mathrm{F}(t)$ and $s^{\mathcal{N}}=t^{\mathcal{N}}$, then $\vdash_{\rho, r}^{\alpha} \Gamma \Rightarrow \Delta, \mathrm{F}(s)$;

(viii) If $\vdash_{\rho, r}^{\alpha} \Gamma, \mathrm{F}(s), \mathrm{F}(t) \Rightarrow \Delta$ and $s^{\mathcal{N}}=t^{\mathcal{N}}$, then $\vdash_{\rho, r}^{\alpha} \Gamma, \mathrm{F}(s) \Rightarrow \Delta$;

(ix) If $\vdash_{\rho, r}^{\alpha} \Gamma \Rightarrow \mathrm{T}(s), \Delta$ and $s^{\mathcal{N}}=t^{\mathcal{N}}$, then $\vdash_{\rho, r}^{\alpha} \Gamma \Rightarrow \mathrm{T}(t), \Delta$;

(x) If $\vdash_{\rho, r}^{\alpha} \Gamma, \mathrm{T}(s) \Rightarrow \Delta$ and $s^{\mathcal{N}}=t^{\mathcal{N}}$, then $\vdash_{\rho, r}^{\alpha} \Gamma, \mathrm{T}(t) \Rightarrow \Delta$;

(xi) If $\vdash_{\rho, r}^{\alpha} \Gamma \Rightarrow \mathrm{F}(s), \Delta$ and $s^{\mathcal{N}}=t^{\mathcal{N}}$, then $\vdash_{\rho, r}^{\alpha} \Gamma \Rightarrow \mathrm{F}(t), \Delta$;

(xii) If $\vdash_{\rho, r}^{\alpha} \Gamma, \mathrm{F}(s) \Rightarrow \Delta$ and $s^{\mathcal{N}}=t^{\mathcal{N}}$, then $\vdash_{\rho, r}^{\alpha} \Gamma, \mathrm{F}(t) \Rightarrow \Delta$.

Lemma 3. (Inversion)

(i) If $\mathrm{SK}^{\infty} \vdash_{\rho, r}^{\alpha} \Gamma \Rightarrow \Delta, A_{0} \wedge A_{1}$, then $\mathrm{SK}^{\infty} \vdash_{\rho, r}^{\alpha} \Gamma \Rightarrow \Delta, A_{i}$ for $i \in\{0,1\}$.

(ii) If $\mathrm{SK}^{\infty} \vdash_{\rho, r}^{\alpha} A \wedge B, \Gamma \Rightarrow \Delta$, then $\mathrm{SK}^{\infty} \vdash_{\rho, r}^{\alpha} A, B, \Gamma \Rightarrow \Delta$.

(iii) If $\mathrm{SK}^{\infty} \vdash_{\rho, r}^{\alpha} \Gamma \Rightarrow \Delta, A \vee B$, then $\mathrm{SK}^{\infty} \vdash_{\rho, r}^{\alpha} \Gamma \Rightarrow \Delta, A, B$.

(iv) If $\mathrm{SK}^{\infty} \vdash_{\rho, r}^{\alpha} A_{0} \vee A_{1}, \Gamma \Rightarrow \Delta$, then $\mathrm{SK}^{\infty} \vdash_{\rho, r}^{\alpha} A_{i}, \Gamma \Rightarrow \Delta$ for $i \in\{0,1\}$.

(v) If $\mathrm{SK}^{\infty} \vdash_{\rho, r}^{\alpha} \Gamma \Rightarrow \Delta, \forall x A$, then $\mathrm{SK}^{\infty} \vdash_{\rho, r}^{\alpha} \Gamma \Rightarrow \Delta, A_{x}(t)$ for each $t \in$ $\mathrm{TERM}_{0}$.

(vi) If $\mathrm{SK}^{\infty} \vdash_{\rho, r}^{\alpha} \exists x A, \Gamma \Rightarrow \Delta$, then $\mathrm{SK}^{\infty} \vdash_{\rho, r}^{\alpha} A_{x}(t), \Gamma \Rightarrow \Delta$ for some $t \in$ TERM $_{0}$.

(vii) If $\mathrm{SK}^{\infty} \vdash_{\rho, 0}^{\alpha} \Rightarrow \mathrm{T}(t)$ and $t^{\mathcal{N}}=\# A$, then $\mathrm{SK}^{\infty} \vdash_{\rho, 0}^{\alpha} \Rightarrow A$.

(viii) If $\mathrm{SK}^{\infty} \vdash_{\rho, 0}^{\alpha} \Rightarrow \mathrm{F}(t)$ and $t^{\mathcal{N}}=\# A$, then $\mathrm{SK}^{\infty} \vdash_{\rho, 0}^{\alpha} \Rightarrow \neg A$.

Proof. As usual on the height $\alpha$ of a derivation.

For the cut-elimination we follow the strategy of removing the topmost cuts of maximal rank.

Theorem 1. (Cut elimination)

For every $\alpha, \beta, \rho, r, n<\omega$ for all $\Gamma, \Delta, A$ with $\operatorname{rk}(A) \leq n$ :

If $\mathrm{SK}^{\infty} \vdash_{\rho, n}^{\alpha} \Gamma \Rightarrow \Delta, A$ and $\mathrm{SK}^{\infty} \vdash_{\rho, n}^{\beta} A, \Gamma \Rightarrow \Delta$, then $\mathrm{SK}^{\infty} \vdash_{\rho, n}^{\alpha \# \beta} \Gamma \Rightarrow \Delta$.

Proof. By use of multiple induction on $\rho, n, \alpha+\beta$. Let $\left(\rho^{\prime}, n^{\prime}, \alpha^{\prime}, \beta^{\prime}\right)<(\rho, n, \alpha, \beta)$ be defined as: $\rho^{\prime}<\rho$, or $\rho^{\prime}=\rho \& n^{\prime}<n$, or $\rho^{\prime}=\rho \& n^{\prime}=n \& \alpha^{\prime}+\beta^{\prime}<\alpha+\beta$.

Assume that the IH holds for all $\left(\rho^{\prime}, n^{\prime}, \alpha^{\prime}, \beta^{\prime}\right)<(\rho, n, \alpha, \beta)$. Then there are the following main cases to consider: (1) at least one of the premises is an axiom, (2) none of the premises is an axiom and $A$ is not principal in at least one of them, and (3) $A$ is principal on both sides.

Case (1) is handled mainly by Lemma 2 . We highlight the case of (TID).

Consider this situation (a case of (1)):

$$
\frac{\Gamma \Rightarrow \mathrm{T}(s), \Delta \quad \Gamma, \mathrm{T}(s) \Rightarrow \mathrm{T}(t), \Delta\left(\text { where } t^{\mathcal{N}}=s^{\mathcal{N}}\right)}{\Gamma \Rightarrow \mathrm{T}(t), \Delta} \text { Lemma 2, (vi) }
$$


Case (2) is standard. Case (3) Consider the following situation where $\forall x A$ is principal in both derivations such that the last inference is an $\forall^{\omega} R$ application of the form

$$
\frac{\ldots \Gamma \Rightarrow \Delta, \forall x A, A_{x}(t) \ldots}{\Gamma \Rightarrow \Delta, \forall x A} \text { for all } t \in \mathrm{TERM}_{0}
$$

And the right premise is derived as (for some $t$ ):

$$
\frac{\forall x A, A_{x}(t), \Gamma \Rightarrow \Delta}{\forall x A, \Gamma \Rightarrow \Delta}
$$

The rest of the argument for this case consists of cutting "diagonally", this means that we first eliminate the occurrences of $\forall x A$ from the context; in this case we can apply the inductive hypothesis because the height is reduced. This procedure results in derivations of the sequents (after possible applications of weakening) $\Gamma \Rightarrow \Delta, A_{x}(t)$, and $A_{x}(t), \Gamma \Rightarrow \Delta$, for all $t \in \mathrm{TERM}_{0}$. The desired end sequent follows, by the fact that $\operatorname{rk}\left(A_{x}(t)\right)<\mathrm{rk}(\forall x A)$.

In the case that $A$ is $\mathrm{T}(t)$ and principal on both sides, derived by (TR) and (TL), so we have

$$
\frac{\vdash_{\rho^{\prime}, r}^{\alpha^{\prime}} \Gamma \Rightarrow B}{\vdash_{\rho, r}^{\alpha} \Gamma \Rightarrow \mathrm{T}(t), \Delta} \quad \frac{\vdash_{\rho^{\prime}, r}^{\beta^{\prime}} B \Rightarrow \Delta}{\vdash_{\rho, r}^{\beta} \Gamma, \mathrm{T}(t) \Rightarrow \Delta} \quad \text { with } \rho^{\prime}<\rho, \alpha^{\prime}<\alpha, \beta^{\prime}<\beta .
$$

Then we can cut directly on $B$ because $B$ has a strictly lower truth rank than $\mathbf{T}(t)$. In this case the upper sequents already have the required shape and so no "diagonal" cut is necessary. Similarly for (FL) and (FR).

\subsection{Strong Kleene fixed-points}

In this subsection we establish the connection between our infinitary proofsystem $\mathrm{SK}^{\infty}$ and the minimal fixed-point of the strong Kleene construction. Kripke suggested partial models in order to construct interpretations of the truth predicate. The construction process is based on a monotone operator $\Lambda: \mathcal{P}(\omega) \rightarrow \mathcal{P}(\omega):$ For $\Phi \subseteq \omega$ we define $n \in \Lambda(\Phi): \Leftrightarrow$

$\left[n \in \mathrm{TRUE}_{0}\right]$ or

$\left[n=\# \mathbf{T}(t)\right.$ for some closed term $t$ and $\left.t^{\mathcal{N}} \in \Phi\right]$ or

$\left[n=\# \mathrm{~F}(t)\right.$ for some closed term $t$ and $\left.\operatorname{neg}(t)^{\mathcal{N}} \in \Phi\right]$ or $t^{\mathcal{N}} \notin$ SENT or

$[n=\# A \wedge B$ for some sentences $A, B$ and $\# A \in \Phi$ and $\# B \in \Phi]$ or

$[n=\# A \vee B$ for some sentences $A, B$ and $(\# A \in \Phi$ or $\# B \in \Phi)]$ or

$[n=\# \forall x A$ for some formula $A$ with the only free variable $x$ and

$\# A_{x}(t) \in \Phi$ for all $\left.t \in \mathrm{TERM}_{0}\right]$ or

[ $n=\# \exists x A$ for some formula $A$ with the only free variable $x$ and

$\# A_{x}(t) \in \Phi$ for some $\left.t \in \mathrm{TERM}_{0}\right]$. 
We arrive at the minimal fixed-point by applying the monotone operator to the empty set and iterating the process:

$$
I_{\Lambda}^{\sigma}:=\Lambda\left(\bigcup_{\tau<\sigma} I_{\Lambda}^{\tau}\right) \quad I_{\Lambda}^{<\sigma}:=\bigcup_{\tau<\sigma} I_{\Lambda}^{\tau} \quad I_{\Lambda}:=\bigcup_{\sigma} I_{\Lambda}^{\sigma}
$$

The minimal fixed-point $I_{\Lambda}$ is the least stage $I_{\Lambda}^{\sigma}$ in this process for which $\Lambda\left(I_{\Lambda}^{\sigma}\right)=$ $I_{\Lambda}^{\sigma}$. We also can define the $\Lambda$ inductive norm $|n|_{\Lambda}:=\min \left\{\sigma \mid n \in I_{\Lambda}^{\sigma}\right\}$ if $n \in I_{\Lambda}$ and $\omega_{1}$ otherwise. It is well known that the minimal fixed-point is only reached at $\omega_{1}^{C K}$ and is $\Pi_{1}^{1}$-complete. ${ }^{4}$

There is an alternative characterization of the fixed-points closer to Kripke's original version as used in the main text of his [13]; although already Kripke points out the possibility of using the $\Lambda$-construction. This makes use of an operator $\Gamma$ such that $\Gamma\left(S_{1}, S_{2}\right)=\left(\left\{\# A:\left(\mathcal{N},\left(S_{1}, S_{2}\right)\right)(A)=\top\right\},\{\# A\right.$ : $\left.\left.\left(\mathcal{N},\left(S_{1}, S_{2}\right)\right)(A)=\perp\right\} \cup \overline{\mathrm{SENT}}\right)$. Both operators give the same fixed-points, i.e.: If $\Gamma\left(S_{1}, S_{2}\right)=\left(S_{1}, S_{2}\right)$, then $\Lambda\left(S_{1}\right)=S_{1}$ and if $\Lambda(S)=S$, then $\Gamma(S, \neg S \cup$ $\overline{\mathrm{SENT}})=(S, \neg S \cup \overline{\mathrm{SENT}})$, where $\neg S:=\{\# A: \# \neg A \in S\} .^{5}$

The connection between the infinitary calculus with the minimal fixed-point models $I_{\Lambda}$ is established in the next lemma:

Lemma 4. If $\mathrm{SK}^{\infty} \vdash_{\rho, 0}^{\alpha} \Rightarrow \Delta$ then $|\# A| \leq \alpha$ for some $A \in \Delta$.

Proof:

By induction on $\alpha$. Let $d$ be a proof of $\Rightarrow \Delta$ in $\mathrm{SK}^{\infty}$ of height $\alpha$.

If the last inference of $d$ is an initial sequent, then it is either (SENT.1) or (Ax.Ar.1). In the first case $t^{\mathcal{N}} \notin \mathrm{SENT}$ and $\# \mathrm{~F}(\mathrm{t}) \in \Lambda(\emptyset)$. In the second case $\Delta$ contains a true arithmetical atom $B$, such that $\# B \in \Lambda(\emptyset)$.

If the last inference is:

(i) (propositional) For $(\mathrm{R} \wedge)$ and $(\mathrm{R} \vee)$ we can use the $\mathrm{IH}$ and one of the corresponding clauses of the definition of $\Lambda$.

(ii) (quantificational) $(\mathrm{R} \exists)$ similar to (ii). For $\left(R \forall^{\omega}\right)$ we have by IH for all $t \in \mathrm{TERM}_{0}$ there is a sentence $B \in \Delta, A_{x}(t)$ with $\# B \in I_{\Lambda}^{\beta}$ for $\beta<\alpha$. If $A \in \Delta$ we get the desired by monotonicity. Otherwise by IH for all $t \in \mathrm{TERM}_{0}$ there is some $\beta<\alpha$ such that $\# A_{x}(t) \in I_{\Lambda}^{\beta}$. But then $\# A_{x}(t) \in I_{\Lambda}^{<\alpha}$ for all $t \in \mathrm{TERM}_{0}$ and then $\forall x A \in I_{\Lambda}^{\alpha}$.

(iii) (truth) For (TR) assume that the last inference is $\frac{\vdash_{\rho, 0}^{\beta} \Rightarrow A}{\vdash_{\rho, 0}^{\alpha} \Rightarrow \mathrm{T}(t), \Delta}$ with $t^{\mathcal{N}}=\# A$ and $\beta<\alpha$. Then we use the IH to argue that $|\# A|=\beta<\alpha$. But then $t^{\mathcal{N}} \in I_{\Lambda}^{<\alpha}$ and $\# \mathrm{~T}(t) \in I_{\Lambda}^{\alpha}$. For $(\mathrm{FR})$ similar.

\footnotetext{
${ }^{4}$ Those results are already mentioned in Kripke [13]. A proof can be found in Burgess [3]. More recently Meadows [14] has given an interesting proof based on an infinitary tableaux.

${ }^{5}$ Compare Halbach [10], section 15.1.
} 
Lemma 5. If $\# A \in I_{\Lambda}^{\alpha}$, then $\mathrm{SK}^{\infty} \vdash_{\rho, 0}^{\alpha+1} \Rightarrow A$, for some $\rho \leq \alpha+1$.

Proof: By induction on $\alpha$. If $\alpha=0$ then $\# A \in \mathrm{TRUE}_{0}$ and $\mathrm{SK}^{\infty} \vdash_{\rho, 0}^{1} \Rightarrow A$ by (Ax.Ar.1). If $\alpha>0$ we have the following cases:

(i) $A=\mathrm{T}(t)$ and $t^{\mathcal{N}} \in I_{\Lambda}^{<\alpha}$, then for some sentence $B, \# B=t^{\mathcal{N}}$ and $\# B \in I_{\Lambda}^{\beta}$ for some $\beta<\alpha$. By IH SK $\vdash_{\rho, 0}^{\beta+1} \Rightarrow B$ and $\mathrm{SK}^{\infty} \vdash_{\rho, 0}^{\alpha+1} \Rightarrow T(t)$ by (TR).

(ii) $A=B \wedge C$ and $\# B \in I_{\Lambda}^{<\alpha}$ and $\# C \in I_{\Lambda}^{<\alpha}$, then there is a $\beta<\alpha$ such that by IH SK $\vdash_{\rho, 0}^{\beta+1} \Rightarrow B$ and $\mathrm{SK}^{\infty} \vdash_{\rho, 0}^{\beta+1} \Rightarrow C$ and then $\mathrm{SK}^{\infty} \vdash_{\rho, 0}^{\alpha+1} \Rightarrow B \wedge C$ by $(\mathrm{R} \wedge)$.

(iii) $A=\forall x B$ and for all $t \in \mathrm{TERM}_{0}, \# B_{x}(t) \in I_{\Lambda}^{<\alpha}$. Then by IH for all $t \in$ $\mathrm{TERM}_{0}$ there is a $\beta_{t}<\alpha$, such that $\mathrm{SK}^{\infty} \vdash_{\rho, 0}^{\beta_{t}+1} \Rightarrow B_{x}(t)$ and $\mathrm{SK}^{\infty} \vdash_{\rho, 0}^{\alpha+1} \Rightarrow$ $\forall x B$ by $\forall^{\omega} \mathrm{R}$.

(iv) -(vi) Other cases $\mathrm{F}(t), \wedge, \exists$ similar.

Corollary 1. $\mathrm{SK}^{\infty} \vdash_{\rho, 0}^{\alpha} \Rightarrow A$ iff $\# A \in I_{\Gamma}$.

\section{Partial logic: a sequent calculus formulation}

We now return from semi-formal systems to formal systems. Our main goal is to present a formalization of PKF. Before we deal with PKF properly we develop its base logic, which is a partial logic, called PAL, in some detail. We give PAL a sequent calculus formulation and prove the cut-elimination theorem. This is accompanied by its soundness and completeness theorems. Towards this end we use a two sided system buildt on a Tait language with finite sets of formulas.

In contrast to the infinitary calculus we now allow for free variables. We follow the convention to distinguish free variables $a, b, c, \ldots$ and bound variables $x, y, z, \ldots ; F V(\Gamma)$ denotes the set of all free variables of $\Gamma$.

\subsection{PAL}

The presentation of the system is structured in: initial sequents, structural, propositional and quantification rules followed by Cut.

Initial Sequent $\quad \Gamma, A \Rightarrow A, \Delta \quad A$ atomic.

\section{Propositional}

$$
\begin{aligned}
& \frac{A, B, \Gamma \Rightarrow \Delta}{A \wedge B, \Gamma \Rightarrow \Delta}(\mathrm{L} \wedge) \quad \frac{\Gamma \Rightarrow \Delta, A \quad \Gamma \Rightarrow \Delta, B}{\Gamma \Rightarrow \Delta, A \wedge B}(\mathrm{R} \wedge) \\
& \frac{A, \Gamma \Rightarrow \Delta \quad B, \Gamma \Rightarrow \Delta}{A \vee B, \Gamma \Rightarrow \Delta}(\mathrm{L} \vee) \quad \frac{\Gamma \Rightarrow \Delta, A, B}{\Gamma \Rightarrow \Delta, A \vee B}(\mathrm{R} \vee)
\end{aligned}
$$




\section{Quantificational}

$$
\begin{aligned}
& \frac{\Gamma, A_{x}(t) \Rightarrow \Delta}{\Gamma, \forall x A \Rightarrow \Delta}(\forall \mathrm{L}) \quad \frac{\Gamma \Rightarrow \Delta, A_{x}(b)}{\Gamma \Rightarrow \Delta, \forall x A}(\forall \mathrm{R}) b \notin F V(\Gamma \cup \Delta) \\
& \frac{\Gamma \Rightarrow \Delta, A_{x}(t)}{\Gamma \Rightarrow \Delta, \exists x A}(\exists \mathrm{R}) \quad \frac{\Gamma, A_{x}(b) \Rightarrow \Delta}{\Gamma, \exists x A \Rightarrow \Delta}(\exists \mathrm{L}) b \notin F V(\Gamma \cup \Delta)
\end{aligned}
$$

\section{Cut}

$$
\frac{\Gamma \Rightarrow \Delta, A \quad A, \Gamma \Rightarrow \Delta}{\Gamma \Rightarrow \Delta}(\mathrm{Cut})
$$

Lemma 6. (Substitution)

If PAL $\vdash \Gamma \Rightarrow \Delta$, then PAL $\vdash \Gamma_{x}(t) \Rightarrow \Delta_{x}(t)$.

The proof proceeds by induction on the height of a derivation with the usual caveat for the rules $(\exists \mathrm{L})$ and $(\forall \mathrm{R})$.

Lemma 7. (Inversion)

All propositional rules and both $(\forall \mathrm{R})$, and $(\exists \mathrm{L})$ are height-preserving invertible.

The proof is by induction on the height of a derivation. It might be noteworthy that in Gentzen's original formulation the rules for left introduction of conjunction and right introduction of disjunction were formulated additively, thus height-preserving inversion of these rules failed.

Some derivable sequents For the connectives $\wedge, \vee$ we can derive the initial sequents of Halbach and Horsten's system: The following sequents are derivable

$$
\begin{aligned}
& A, B \Rightarrow A \wedge B \\
& A \wedge B \Rightarrow A \\
& A \wedge B \Rightarrow B \\
& A \vee B \Rightarrow A, B \\
& A \Rightarrow A \vee B \\
& B \Rightarrow A \vee B
\end{aligned}
$$

And also $(\neg \neg L) \quad \neg \neg A \Rightarrow A$ and $(\neg \neg R) \quad A \Rightarrow \neg \neg A$ are derivable (by use of the equivalences stated above). Furthermore, the following rule is admissible in PAL (again by use of the equivalences stated above):

$$
\frac{\Gamma \Rightarrow \Delta}{\neg \Delta \Rightarrow \neg \Gamma}(\text { Contra })
$$


It follows from these facts that if a sequent is derivable in Halbach and Horsten's system, then it also derivable in PAL. The other direction can also be established. The proof is by induction on the height of a derivation. Clearly, $\mathrm{PAL} \vdash A, \Gamma \Rightarrow \Delta, A$. We outline the case for $(\mathrm{L} \wedge)$. The remaining rules, i.e. $(\mathrm{R} \wedge),(\mathrm{LV})$, and $(\mathrm{R} \vee)$ are similar.

$$
\begin{gathered}
\frac{\frac{A \wedge B \Rightarrow A}{A \wedge B, \Gamma \Rightarrow \Delta, A} \quad A, B, \Gamma, \Rightarrow \Delta}{\frac{A \wedge B \Rightarrow B}{A \wedge B, \Gamma \Rightarrow \Delta, B}} \\
\frac{\frac{A \wedge B, B, \Gamma, \Gamma \Rightarrow \Delta, \Delta}{B, A \wedge B, \Gamma \Rightarrow \Delta}}{A \wedge B, \Gamma \Rightarrow \Delta, \Delta} \\
\frac{}{A \wedge B, \Gamma \Rightarrow \Delta}
\end{gathered}
$$

The system PAL has, however, the advantage of enjoying the cut-elimination theorem. This is established next.

Theorem 2 (Cut-elimination). For every derivation of an end sequent in PAL with an application of cut there is a derivation in PAL without an application of cut with the same end sequent.

We follow the strategy of removing the topmost maximal rank cuts by use of induction on the rank of a cut with a subinduction on the height of the derivation. Then there are the following main cases to consider: (1) at least one of the premises is an axiom, (2) none of the premises is an axiom and the cut-formula is not principal in at least one of them, and (3) the cut-formula is principal on both sides.

By entertaining the usual definition of subformulas and consistency the next corollary is immediate.

\section{Corollary 2.}

(i) (Subformula) If a sequent has a cut-free PAL-derivation, then every formula occurring in the derivation is a subformula of a formula in the endsequent of it.

(ii) (Consistency) The empty sequent is not derivable in PAL.

\subsection{Semantic: Soundness and Completeness}

A partial model $\mathfrak{M}$ is $(M, \mathcal{I})$, where $M \neq \emptyset$ is the domain and $\mathcal{I}$ is a partial interpretation. We only treat predicates as partial, i.e. constants and functions are defined as usual. ${ }^{6}$

As we are working in a Tait language we assume that for every predicate $\mathrm{R}$ we have a 'complement' $\bar{R}$. Predicates $\mathrm{R}$ and their counterparts $\bar{R}$ are both interpreted by an ordered pair of relations which are neither required to be

\footnotetext{
${ }^{6}$ We follow Blamey's [1] presentation.
} 
disjoint nor exhaustive. So we have for every model $\mathcal{M}$ the extension of $\mathrm{R}$, which is denoted by $\mathrm{R}_{+}^{\mathfrak{M}}$, as well as the antiextension of $\mathrm{R}, \mathrm{R}_{-}^{\mathfrak{M}}$, the extension of $\bar{R}$, which is $\bar{R}_{+}^{\mathfrak{M}}$, and the antiextension of $\bar{R}$, which is $\bar{R}_{-}^{\mathfrak{M}}$. In order for $\bar{R}$ to be the complement of $\mathrm{R}$ we require that $\mathrm{R}_{+}^{\mathfrak{M}}=\bar{R}_{-}^{\mathfrak{M}}$ and $\mathrm{R}_{-}^{\mathfrak{M}}=\bar{R}_{+}^{\mathfrak{M}}$.

In contrast to classical logic where the extension of $\mathrm{R}$ is sufficient to define all others we have here more possibilities. The fact that the extension and antiextension do not have to be exhaustive simulates the partiality. Without the restriction that the extension of $\mathrm{R}$ and $\bar{R}$ have to be disjoint we allow for gluts as well.

For a term $t$, a model $\mathfrak{M}$ and an assignment $s$, we define $t^{\mathfrak{M}_{s}}$ as usual.

Definition 1. Let $\mathfrak{M}$ be a partial model and $s$ a variable assignment. ${ }^{7}$

$$
\begin{aligned}
& \mathfrak{M}_{s}\left(\mathrm{R} t_{1}, \ldots t_{n}\right)= \begin{cases}\top, & \text { iff }\left\langle t_{1}^{\mathfrak{M}_{s}}, \ldots, t_{n}^{\mathfrak{M}_{s}}\right\rangle \in \mathrm{R}_{+}^{\mathfrak{M}} \\
\perp, & \text { iff }\left\langle t_{1}^{\mathfrak{M}_{s}}, \ldots, t_{n}^{\mathfrak{M}_{s}}\right\rangle \in \mathrm{R}_{-}^{\mathfrak{M}} \\
*, & \text { otherwise }\end{cases} \\
& \mathfrak{M}_{s}\left(\bar{R} t_{1}, \ldots t_{n}\right)= \begin{cases}\top, & \text { iff }\left\langle t_{1}^{\mathfrak{M}_{s}}, \ldots, t_{n}^{\mathfrak{M}_{s}}\right\rangle \in \bar{R}_{+}^{\mathfrak{M}} \\
\perp, & \text { iff }\left\langle t_{1}^{\mathfrak{M}_{s}}, \ldots, t_{n}^{\mathfrak{M}_{s}}\right\rangle \in \bar{R}_{-}^{\mathfrak{M}} \\
*, & \text { otherwise }\end{cases}
\end{aligned}
$$

The connectives follow the truth tables for strong Kleene logic and the quantifiers are interpreted as usual..

By induction on the build up of $A$ we can show that also for defined negation it follows the usual pattern of many valued logic, i.e.:

$$
\mathfrak{M}_{s}(\neg A)= \begin{cases}\top, & \text { iff } \mathfrak{M}_{s}(A)=\perp \\ \perp, & \text { iff } \mathfrak{M}_{s}(A)=\top \\ *, & \text { iff } \mathfrak{M}_{s}(A)=*\end{cases}
$$

In order to have a notion of a sequence being modeled we follow Blamey in requiring truth preservation and falsity anti-preservation.

Definition 2. ( $\mathfrak{M}, s)$ models $\Gamma \Rightarrow \Delta$ iff

(i) if $\mathfrak{M}_{s}(A)=\top$ for all $A \in \Gamma$ then there is a $B \in \Delta$ with $\mathfrak{M}_{s}(B)=\top$; and

(ii) if $\mathfrak{M}_{s}(B)=\perp$ for all $B \in \Delta$, then there is an $A \in \Gamma$ with $\mathfrak{M}_{s}(A)=\perp$.

Let $\mathcal{M}$ be some class of partial models, then $\Gamma \models_{\mathcal{M}} \Delta$ iff every model in $\mathcal{M}$ models $\Gamma \Rightarrow \Delta$. Let $\Sigma$ be a countable set of sequents, then $(\mathfrak{M}, s)$ is a model of $\Sigma$ iff $(\mathfrak{M}, s)$ models all sequents in $\Sigma$. $\mathcal{K}(\Sigma)$ is the class of all models of $\Sigma$. $\Gamma \vdash_{\Sigma} \Delta: \Leftrightarrow$ there is a finite set of sequents $\Sigma_{0} \subseteq \Sigma$ such that $\Sigma_{0} \vdash \Gamma \Rightarrow \Delta$.

With these notions at hand soundness is established as usual.

Theorem 3. (Soundness) Let $\Sigma$ be a countable set of sequents. If $\Gamma \vdash_{\Sigma} \Delta$, then $\Gamma \models \mathcal{K}(\Sigma) \Delta$.

\footnotetext{
${ }^{7}$ Equivalently we can think of an interpretation of an $m$-ary relation symbol $\mathrm{R}$ as a single function $M^{m} \rightarrow\{0,1, n, b\}$ where $n$ stands for neither and $b$ stands for both. With this we can connect it to the usual version of the four valued logic FDE, or BDM. See for example Priest [15], chapter 8 and Field [7].
} 
For the Completeness result one can follow Buss' [4] proof search method or adopt Blamey's result in [1].

Theorem 4. (Completeness) Let $\Sigma$ be a countable set of sequents. If $\Gamma \models_{\mathcal{K}(\Sigma)} \Delta$, then $\Gamma \vdash_{\Sigma} \Delta$.

\section{PKF: Adding compositional truth sequents to PAL}

Let's call the extension of PAL by initial sequents of identity, arithmetic and truth PKF. ${ }^{8}$ Since we work with a two-sided sequent calculus with a special treatment of negation, the usual initial sequents will come in two versions.

The main goal of the next section is to establish an embedding of PKF in $\mathrm{SK}^{\infty}$. But let us commence with the formulation of PKF followed by establishing some mathematical properties thereof.

\subsection{Presentation of PKF}

This system is an extension of the positive partial logic. It contains additional initial axioms for equality, initial sequents for the axioms of Peano Arithmetic, a rule of induction, and finally initial sequents for the truth predicate.

\subsubsection{Initial sequents for equality and inequality}

(E1R) $\quad \Gamma \Rightarrow t=t, \Delta$

(E1L) $\quad \Gamma, t \neq t \Rightarrow \Delta$

(E2R) $\quad \Gamma, s=t, A_{x}(s) \Rightarrow A_{x}(t), \Delta$

(E2L) $\quad \Gamma, \neg A_{x}(t) \Rightarrow \neg s=t, \neg A_{x}(s), \Delta$

\subsubsection{Initial sequents for Peano Arithmetic}

The formal system PKF contains the Peano Arithmetic, PA. Due to our formulation of the system as a whole the axioms of PA come in a left version and a right version, i.e. for all axioms $A$ of PA (without induction):

$\left(\mathrm{PA} \mathrm{R}_{\mathrm{R}}\right.$

$\left(\mathrm{PA} A_{\mathrm{L}}\right)$

$$
\begin{aligned}
& \Gamma \Rightarrow A, \Delta \\
& \Gamma, \neg A \Rightarrow \Delta
\end{aligned}
$$

Instead of using the (schema) of induction, the following rule is adopted:

$$
\frac{A_{x}(b), \Gamma \Rightarrow \Delta, A_{x}(b+1)}{A_{x}(\underline{0}), \Gamma \Rightarrow \Delta, A_{x}(t)}(\text { Ind })
$$

Where $t$ is any term, $A$ any formula of the language, $b$ must not occur freely in $A_{x}(\underline{0}), \Gamma, \Delta$.

\footnotetext{
${ }^{8}$ Halbach and Horsten coined the system in [11] PKF; our system is a variation thereof.
} 


\subsubsection{Initial sequents for Truth}

We present the initial sequents of truth for PKF.

(PKF1R)

(PKF1L)

(PKF2R)

(PKF2L)

(PKF3R)

(PKF3L)

(PKF4R)

(PKF4L)

(PKF5R)

(PKF5L)

(PKF6R)

(PKF6L)

(PKF7R)

(PKF7L)

(PKF8R)

(PKF8L)

(PKF9R)

(PKF9L)

(PKF10R)

(PKF10L)

$$
\begin{aligned}
& \Gamma, \operatorname{ct}(a), \operatorname{ct}(b), \operatorname{val}(a)=\operatorname{val}(b) \Rightarrow \mathrm{T}(\operatorname{id}(a, b)), \Delta \\
& \Gamma, \operatorname{ct}(a), \operatorname{ct}(b), \mathrm{T}(\operatorname{id}(a, b)) \Rightarrow \operatorname{val}(a)=\operatorname{val}(b), \Delta \\
& \Gamma, \operatorname{ct}(a), \operatorname{ct}(b), \operatorname{val}(a) \neq \operatorname{val}(b) \Rightarrow \mathrm{F}(\operatorname{id}(a, b)), \Delta \\
& \Gamma, \operatorname{ct}(a), \operatorname{ct}(b), \mathrm{F}(\operatorname{id}(a, b)) \Rightarrow \operatorname{val}(a) \neq \operatorname{val}(b), \Delta
\end{aligned}
$$

$$
\begin{aligned}
& \Gamma, \operatorname{ct}(b), \mathrm{T}(\operatorname{val}(b)) \Rightarrow \mathrm{T}(\operatorname{tr}(b)), \Delta \\
& \Gamma, \operatorname{ct}(b), \mathrm{T}(\operatorname{tr}(b)) \Rightarrow \mathrm{T}(\operatorname{val}(b)), \Delta \\
& \Gamma, \operatorname{ct}(b), \mathrm{F}(\operatorname{val}(b)) \Rightarrow \mathrm{T}(\operatorname{ntr}(b)), \Delta \\
& \Gamma, \operatorname{ct}(b), \mathrm{T}(\operatorname{ntr}(b)) \Rightarrow \mathrm{F}(\operatorname{val}(b)), \Delta
\end{aligned}
$$

$\Gamma, \operatorname{sent}(a), \operatorname{sent}(b), \mathrm{T}(a) \wedge \mathrm{T}(b) \Rightarrow \mathrm{T}($ and $(s, t)), \Delta$

$\Gamma, \operatorname{sent}(a), \operatorname{sent}(b), \mathrm{T}(\operatorname{and}(a, b)) \Rightarrow \mathrm{T}(a) \wedge \mathrm{T}(b), \Delta$

$\Gamma, \operatorname{sent}(a), \operatorname{sent}(b), \mathrm{T}(a) \vee \mathrm{T}(b) \Rightarrow \mathrm{T}($ or $(a, b)), \Delta$

$\Gamma, \operatorname{sent}(a), \operatorname{sent}(b), \mathrm{T}(\operatorname{or}(a, b)) \Rightarrow \mathrm{T}(a) \vee \mathrm{T}(b), \Delta$

$\Gamma, \operatorname{var}(v)$, form $(a, v), \forall x \mathrm{~T}(a(\dot{x})) \Rightarrow \mathrm{T}($ all $(v, a)), \Delta$

$\Gamma, \operatorname{var}(v), \operatorname{form}(a, v), \mathrm{T}(\operatorname{all}(v, a)) \Rightarrow \forall x \mathrm{~T}(a(\dot{x})), \Delta$

$\Gamma, \operatorname{var}(v)$, form $(a, v), \exists x \mathrm{~T}(a(\dot{x})) \Rightarrow \mathrm{T}(\operatorname{ex}(v, a)), \Delta$

$\Gamma, \operatorname{var}(v)$, form $(a, v), \mathrm{T}(\operatorname{ex}(v, a)) \Rightarrow \exists x \mathrm{~T}(a(\dot{x})), \Delta$

$\Gamma, \operatorname{sent}(t), \mathrm{F}(t) \Rightarrow \mathrm{T}(\mathrm{neg}(t)), \Delta$

$\Gamma, \operatorname{sent}(t), \mathrm{T}(\operatorname{neg}(t)) \Rightarrow \mathrm{F}(t), \Delta$

$\Gamma, \mathrm{T}(a) \Rightarrow \operatorname{sent}(a), \Delta$

$\Gamma, \neg \operatorname{sent}(a) \Rightarrow \mathrm{F}(a), \Delta$

This completes the presentation of PKF. The system is intended to include a one-sided Tait system as well as its negated counterpart. The following sequents 
completing this picture are derivable in PKF:

$$
\begin{aligned}
& \operatorname{ct}(a), \operatorname{ct}(b), \operatorname{val}(a) \neq \operatorname{val}(b) \Rightarrow T(\operatorname{nid}(a, b)) \\
& \operatorname{ct}(a), \operatorname{ct}(b), T(\operatorname{nid}(a, b)) \Rightarrow \operatorname{val}(a) \neq \operatorname{val}(b) \\
& \operatorname{ct}(a), \operatorname{ct}(b), \operatorname{val}(a)=\operatorname{val}(b) \Rightarrow \mathrm{F}(\operatorname{nid}(a, b)) \\
& \operatorname{ct}(a), \operatorname{ct}(b), \mathrm{F}(\operatorname{nid}(a, b)) \Rightarrow \operatorname{val}(a)=\operatorname{val}(b) \\
& \operatorname{sent}(a), \operatorname{sent}(b), \neg(T(a) \wedge T(b)) \Rightarrow \mathrm{F}(\operatorname{and}(s, t)) \\
& \operatorname{sent}(a), \operatorname{sent}(b), \mathrm{F}(\operatorname{and}(a, b)) \Rightarrow \neg(T(a) \wedge T(b)) \\
& \operatorname{sent}(a), \operatorname{sent}(b), \mathrm{F}(a) \wedge \mathrm{F}(b) \Rightarrow \mathrm{F}(\operatorname{or}(a, b)) \\
& \operatorname{sent}(a), \operatorname{sent}(b), \mathrm{F}(\operatorname{or}(a, b)) \Rightarrow \mathrm{F}(a) \wedge \mathrm{F}(b) \\
& \operatorname{var}(v), \text { form }(a, v), \exists x \mathrm{~F}(a(\dot{x})) \Rightarrow \mathrm{F}(\operatorname{all}(v, a)) \\
& \operatorname{var}(v), \text { form }(a, v), \mathrm{F}(\operatorname{all}(v, a)) \Rightarrow \exists x \mathrm{~F}(a(\dot{x})) \\
& \operatorname{var}(v), \operatorname{form}(a, v), \mathrm{F}(\operatorname{ex}(v, a)) \Rightarrow \forall x \mathrm{~F}(a(\dot{x})) \\
& \operatorname{var}(v), \operatorname{form}(a, v), \forall x \mathrm{~F}(a(\dot{x})) \Rightarrow \mathrm{F}(\operatorname{ex}(v, a)) \\
& \operatorname{sent}(t), \mathrm{F}(\operatorname{neg}(t)) \Rightarrow \mathrm{T}(t) \\
& \operatorname{sent}(t), \mathrm{T}(t) \Rightarrow \mathrm{F}(\operatorname{neg}(t)) \\
& \operatorname{ct}(b), \mathrm{F}(\operatorname{val}(b)) \Rightarrow \mathrm{F}(\operatorname{tr}(b)) \\
& \operatorname{ct}(b), \mathrm{F}(\operatorname{tr}(b)) \Rightarrow \mathrm{F}(\operatorname{val}(b))
\end{aligned}
$$

The proofs make use of the fact that in PKF we have the rules (PKF9R), (PKF9L) for commuting truth and negation and that for the syntactic predicates we can make use of the usual interdefinabilites. As an example we sketch $(\mathrm{j})$ :

$$
\begin{aligned}
\operatorname{var}(v), \text { form }(a, v), \mathrm{F}(\operatorname{all}(v, a)) & \Rightarrow \mathrm{T}(\operatorname{neg}(\operatorname{all}(v, a))) \\
\operatorname{var}(v), \text { form }(a, v), \mathrm{T}(\operatorname{neg}(\operatorname{all}(v, a))) & \Rightarrow \mathrm{T}(\operatorname{ex}(\operatorname{neg}(v, a)) \\
\operatorname{var}(v), \text { form }(a, v), \mathrm{T}(\operatorname{ex}(\operatorname{neg}(v, a)) & \Rightarrow \exists x \mathrm{~T}(\operatorname{neg}(a(\dot{x})) \\
\operatorname{var}(v), \text { form }(a, v), \exists x \mathrm{~T}(\operatorname{neg}(a(\dot{x})) & \Rightarrow \exists x \mathrm{~F}(a(\dot{x}))
\end{aligned}
$$

Lemma 8. PKF $\vdash A_{x}(s) \Rightarrow A_{x}(t)$, if $s^{\mathcal{N}}=t^{\mathcal{N}}$, for any $A$.

Making use of (a)-(n) we get the following:

Lemma 9 (Contraposition). The rule of contraposition (Cont) is admissible in PKF,

$$
\frac{\Gamma \Rightarrow \Delta}{\neg \Delta \Rightarrow \neg \Gamma} \text { (Cont) }
$$

With this lemma we see that our system is equivalent to the system presented in [11].

Lemma 10. If $\Rightarrow A, \neg A$ is derivable in $\mathrm{PKF}$, then the following rules are admissible in PKF:

$$
\frac{A, \Gamma \Rightarrow \Delta}{\Gamma \Rightarrow \Delta, \neg A} \quad \frac{\Gamma \Rightarrow \Delta, A}{\neg A, \Gamma \Rightarrow \Delta}
$$


The proof is straightforward.

Lemma 11. $\Rightarrow A, \neg A$ is derivable in PKF given that $A$ is arithmetical.

Proof by induction on the complexity of $A$.

Corollary 3. If $A$ is arithmetical, then the following rules are admissible for PKF :

$$
\frac{A, \Gamma \Rightarrow \Delta}{\Gamma \Rightarrow \Delta, \neg A} \quad \frac{\Gamma \Rightarrow \Delta, A}{\neg A, \Gamma \Rightarrow \Delta}
$$

Corollary 4. PA restricted to the language of Peano Arithmetic is a subsystem of PKF.

The proof is established as a direct consequence of the above lemmas and corollaries.

\subsection{Adequacy of PKF}

We want to restate a known fact about PKF, i.e.:

\section{Lemma 12.}

(i) PKF $\vdash A\left(a_{1}, \ldots, a_{n}\right) \Rightarrow \mathrm{T}\left(\left\ulcorner A \dot{a}_{1} \ldots \dot{a}_{n}\right\urcorner\right)$.

(ii) $\mathrm{PKF} \vdash \mathrm{T}\left(\left\ulcorner A \dot{a}_{1} \ldots \dot{a}_{n}\right\urcorner\right) \Rightarrow A\left(a_{1}, \ldots, a_{n}\right)$

The proof proceeds by induction on the complexity of $A$ and it follows closely along the lines of Halbach \& Horsten 2006 [11], Halbach 2014 [10] (Theorem 16.11), p.227ff. In the case, where $A$ is atomic, we can use the initial sequents PKF1 - PKF4 or $(a)$ and $(b)$. In the inductive case, we use the initial sequents PKF5 - PKF8, or $(e)-(l)$.

In Fischer et al. [8] a criterion of adequacy for axiomatic theories trying to capture a semantic theory was discussed. There it was already mentioned that PKF is adequate with respect to the class of strong Kleene fixed-points based on the standard interpretation of arithmetic. ${ }^{9}$ We repeat the argument here for our version of PKF.

We use $(\mathcal{N}, S) \models$ PKF short for all sequents derivable in PKF are modeled by a model in which the arithmetical vocabulary is interpreted by the standard model of arithmetic and the extension for the truth predicate is $S$ whereas the antiextension is given by $\bar{S}:=\{\# A: \# \neg A \in S\} \cup \overline{\mathrm{SENT}}$.

\section{Theorem 5.}

$$
(\mathcal{N},(S, \bar{S})) \models \text { PKF iff } \Lambda(S)=S .
$$

\footnotetext{
${ }^{9}$ This observation is due to Johannes Stern.
} 
Proof (Sketch): The right to left direction is soundness and was already established in Halbach \& Horsten [11] for their system. The proof is tedious but straightforward.

The other direction is a simple consequence of Lemma 12 . Let $(\mathcal{N},(S, \bar{S})) \models$ PKF. Then $\# A \in S$ iff $(\mathcal{N},(S, \bar{S})) \models \Rightarrow \mathrm{T}(\ulcorner\mathrm{A}\urcorner)$ iff $(\mathcal{N},(S, \bar{S})) \models \Rightarrow A$ iff $\# A \in \Lambda(S)$.

Remark. In the second to last equivalence we used the fact that by Lemma 12 $A \Rightarrow \mathrm{T}(\ulcorner\mathrm{A}\urcorner)$ and $\mathrm{T}(\ulcorner\mathrm{A}\urcorner) \Rightarrow \mathrm{A}$ are derivable in $\mathrm{PKF}$.

\section{Embedding of PKF in $\mathrm{SK}^{\infty}$}

In the following we will establish an embedding of PKF in our infinitary system $\mathrm{SK}^{\infty}$. At this point we only consider the height of the derivations and therefore we can simplify the presentation by taking $\mathrm{SK}^{\infty} \vdash^{\alpha} \Gamma \Rightarrow \Delta$ as an abbreviation of $\mathrm{SK}^{\infty} \vdash \vdash_{\rho, r}^{\alpha} \Gamma \Rightarrow \Delta$ for some $\rho, r$.

Theorem 6 (Embedding of PKF in $\mathrm{SK}^{\infty}$ ).

If $\mathrm{PKF} \vdash^{k} \Gamma \Rightarrow \Delta$, with the free variables $a_{1}, \ldots, a_{n}$, then

$\mathrm{SK}^{\infty} \vdash^{\omega \cdot k} \Gamma \Rightarrow \Delta\left(a_{1} / t_{1}, \ldots, a_{n} / t_{n}\right)$ for all $t_{i} \in \mathrm{TERM}_{0} i \leq n$.

The proof proceeds by induction on the height of a derivation. Let us first note that the equality axioms of $\mathrm{PKF}$ are derivable in $\mathrm{SK}^{\infty}$; and furthermore that all initial sequents of Peano Arithmetic are derivable in $\mathrm{SK}^{\infty}$ as well. Moreover, both the structural and the propositional rules are structurally identical in both systems. The main work is on

- Initial sequents for truth, and

- Induction (Ind) and universal quantification.

Let us start with derivations of some initial sequents for truth.

Derivation of $(\mathrm{PKF1R})$, i.e. $\Gamma, \operatorname{ct}(a), \operatorname{ct}(b), \operatorname{val}(a)=\operatorname{val}(b) \Rightarrow \mathrm{T}(\mathrm{id}(\mathrm{a}, \mathrm{b})), \Delta$. Let $s, t \in \mathrm{TERM}_{0}$. Then either $s^{\mathcal{N}}=t^{\mathcal{N}}$ or $s^{\mathcal{N}} \neq t^{\mathcal{N}}$. In the first case

$\mathrm{SK}^{\infty} \vdash \Rightarrow \operatorname{val}(s)=\operatorname{val}(t)$ by $\left(\mathrm{PA}_{\mathrm{R}}\right)$ and with $(\mathrm{TR})$ and weakening we get the desired. Similar for the other case.

Derivation of (PKF3R), i.e. $\operatorname{ct}(b), \mathrm{T}(b) \Rightarrow \mathrm{T}(\operatorname{tr}(b))$ in $\mathrm{SK}^{\infty}$. If $t \notin \mathrm{TERM}_{0}$, then $\mathrm{SK}^{\infty} \vdash^{1} \mathrm{ct}(t) \Rightarrow$. Let $t \in \mathrm{TERM}_{0}$, then $\operatorname{tr}(t)^{\mathcal{N}}=\# \mathbf{T}(t)$ and

$$
\frac{\mathrm{T}(t) \Rightarrow \mathrm{T}(t)}{\mathrm{T}(t) \Rightarrow \mathrm{T}(\operatorname{tr}(t))}
$$

Similarly, for (PKF3L), (PKF4R), (PKF4L) with contraposition. Derivation of $\left(\right.$ PKF5R), i.e. $\operatorname{sent}(a), \operatorname{sent}(b), \mathrm{T}(a) \wedge \mathrm{T}(b) \Rightarrow \mathrm{T}($ and $(a, b))$ in $\mathrm{SK}^{\infty}$. For this end, we assume $s, t \in \mathrm{TERM}_{0}$. If either $s$ or $t$ are not in SENT, then we get sent $(s), \operatorname{sent}(t),(\mathrm{T}(s) \wedge \mathrm{T}(t)) \Rightarrow \mathrm{T}($ and $(s, t))$ by $\left(\mathrm{PA}_{\mathrm{L}}\right)$. If both are sentences say $s^{\mathcal{N}}=\# A$, and $t^{\mathcal{N}}=\# B$ we argue as follows: 


$$
\frac{\frac{A \Rightarrow A}{\mathrm{~T}(s), \mathrm{T}(t) \Rightarrow A}(\mathrm{TL}) \quad \frac{B \Rightarrow B}{\mathrm{~T}(s), \mathrm{T}(t) \Rightarrow B}}{\frac{\mathrm{T}(s), \mathrm{T}(t) \Rightarrow A \wedge B}{\mathrm{~T}(s), \mathrm{T}(t) \Rightarrow \mathrm{T}(\operatorname{and}(s, t))}(\mathrm{TR})}
$$

Next we want to derive (PKF8R), i.e. $\operatorname{var}(v)$, form $(a, v), \forall x \mathrm{~T}(\mathrm{ax}) \Rightarrow \mathrm{T}(\mathrm{all}(\mathrm{b}, \mathrm{a}))$. Let $r, s$ be in TERM $\mathrm{T}_{0}$. If it is not the case that $r^{\mathcal{N}}$ is a formula with one free variable or $s^{\mathcal{N}}$ is not a bound variable, then $\operatorname{sent}($ all $(s, r)) \Rightarrow$ is in $\left(\mathrm{PA}_{\mathrm{L}}\right)$. If it is the case that $r^{\mathcal{N}}=\# A_{x}(b)$ is a formula with one free variable and $s^{\mathcal{N}}=\# x$ is a bound variable, then since $A_{x}(t) \Rightarrow A_{x}(t)$ is derivable in $\mathrm{SK}^{\infty}$ for all $t \in \mathrm{TERM}_{0}$ we get by $(\mathrm{TL})$ that $\mathrm{T}(r \dot{t}) \Rightarrow A_{x}(t)$ for every $t \in \mathrm{TERM}_{0}$ and by $(\forall \mathrm{L})$ we get $\forall x \mathrm{~T}(r \dot{x}) \Rightarrow A_{x}(t)$ for every $t \in \mathrm{TERM}_{0}$. Then we can use $(\forall \mathrm{R})$ to get $\forall x \mathrm{~T}(r \dot{x}) \Rightarrow \forall x A$ for any bound variable $x$. So with (TR) also $\forall x \mathrm{~T}(r \dot{x}) \Rightarrow \mathrm{T}($ all $(\mathrm{s}, \mathrm{r}))$.

Next we show (PKF9R). We argue by distinction of cases. For $t \notin$ SENT we get the instance by $\left(\mathrm{PA} A_{\mathrm{L}}\right)$. In the other case there is a sentence $A$ with $t^{\mathcal{N}}=\# A$.

Then we can argue as follows: $\frac{\frac{\neg A \Rightarrow \neg A}{\mathrm{~F}(t) \Rightarrow \neg A}(\mathrm{FL})}{\mathrm{F}(t) \Rightarrow \mathrm{T}(\operatorname{neg}(t))}(\mathrm{TR})$

(PKF9L) similar.

This shows that for all initial sequents $\Gamma \Rightarrow \Delta$ of PKF, there is some $k<\omega$, such that $\mathrm{SK}^{\infty} \vdash^{k} \Gamma \Rightarrow \Delta$.

For all the rules except $(\forall \mathrm{R}),(\exists \mathrm{L})$ and (Ind) we can argue directly by IH. For $(\forall \mathrm{R})$ we argue (following Buchholz [2] which in turn is based on Schütte's method) as follows: If the last inference is

$$
\frac{\mathrm{PKF} \vdash k^{\prime} \Gamma \Rightarrow \Delta, A_{x}(b)}{\mathrm{PKF} \vdash^{k} \Gamma \Rightarrow \Delta, \forall x A}(\forall \mathrm{R}) b \notin F V(\Gamma \cup \Delta) \text { with } k^{\prime}<k \text {. }
$$

Then by IH we have for all $t \in \mathrm{TERM}_{0}, \mathrm{SK}^{\infty} \vdash \omega \cdot k^{\prime} \Gamma \Rightarrow \Delta, A_{x}(t)$ and $\omega \cdot k=\sup \left\{\omega \cdot k^{\prime}+1\right\}$.

For (Ind) let $\frac{\mathrm{PKF} \vdash^{k^{\prime}} A_{x}(b), \Gamma \Rightarrow \Delta, A_{x}(b+1)}{\mathrm{PKF} \vdash^{k} A_{x}(\underline{0}), \Gamma \Rightarrow \Delta, A_{x}(t)}$

By IH we can assume that $\mathrm{SK}^{\infty} \vdash^{\omega \cdot k^{\prime}} A_{x}(s), \Gamma \Rightarrow \Delta, A_{x}(s+1)$ for all $s \in \mathrm{TERM}_{0}$. But then for every $t \in \mathrm{TERM}_{0}$ there is an $n \in \mathbb{N}$ such that $t^{\mathcal{N}}=n$ and by $n$

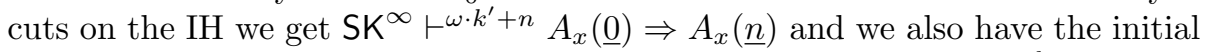
sequent $A_{x}(\underline{n}) \Rightarrow A_{x}(t)$. So by a cut we get the desired $\mathrm{SK}^{\infty} \vdash^{\omega \cdot k} A_{x}(\underline{0}) \Rightarrow$ $A_{x}(t)$.

\section{An upper bound for PKF theorems}

In this section we want to make use of the embedding to show that all the theorems provable in PKF will appear in the fixed-point construction already at a level less than $\omega^{\omega}$. 
Theorem 7. If $\mathrm{PKF} \vdash \Rightarrow A$, then $\# A \in I_{\Lambda}^{\omega^{\omega}}$.

Proof: If PKF $\vdash \Rightarrow A$, then by symmetry, i.e. by Lemma 12 , we know that $\mathrm{PKF} \vdash \Rightarrow \mathrm{T}(\ulcorner A\urcorner)$.

The only rule that includes nonatomic formulas in a crucial way is the induction rule. However induction is formulated in a way that it can be replaced by a schema of internal induction: $\frac{\Gamma, \mathrm{T}(\ulcorner A(\dot{x})\urcorner) \Rightarrow \mathrm{T}(\ulcorner A(x \dot{+} 1)\urcorner), \Delta}{\Gamma, \mathrm{T}(\ulcorner A(\underline{0})\urcorner) \Rightarrow \mathrm{T}(\ulcorner A(t)\urcorner), \Delta}$. Call the system with internal induction PKFINT. Then it is easy to show with symmetry, i.e. by Lemma 12, that

Lemma 13. If $\mathrm{PKF} \vdash \Gamma \Rightarrow \Delta$, then $\mathrm{PKF}_{\mathrm{INT}} \vdash \Gamma \Rightarrow \Delta$.

The system PKF INT has the nice feature that all the nonlogical axioms and rules are based on literals. This allows us to apply standard cut elimination techniques in order to reduce the cuts to cuts on literals $\vdash_{l i t}{ }^{10}$

Lemma 14. If $\mathrm{PKF}_{\mathrm{INT}} \vdash_{m}^{l} \Gamma \Rightarrow \Delta$, then $\mathrm{PKF}_{\mathrm{INT}} \vdash_{l i t}^{2_{m}^{l}} \Gamma \Rightarrow \Delta$.

Using Lemma 13 we can conclude that $\mathrm{PKF}_{\mathrm{INT}} \vdash \Rightarrow \mathrm{T}(\ulcorner A\urcorner)$ and with Lemma 14 we can show that there is some $k<\omega$ such that $\mathrm{PKF}_{\mathrm{INT}} \vdash_{\text {lit }}^{k} \Rightarrow \mathrm{T}(\ulcorner A\urcorner)$.

Then we can use our embedding to show that then $\mathrm{SK}^{\infty} \vdash_{\text {lit }}^{\omega^{k}} \Rightarrow \mathrm{T}(\ulcorner A\urcorner)$.

Although we get a full cut elimination in our infinitary system we cannot make use of it for a succinct estimation of the upper bound. Therefore, we employ an asymmetric interpretation used by Cantini [6] and slightly modify it for our system.

We define $\models A[\beta, \delta]$ by induction on the build up of $A$ with the idea being that all the positive occurrences are interpreted at the level $I_{\Lambda}^{\delta}$, whereas the negative occurrences are interpreted at the level $I_{\Lambda}^{\beta}$ so $\models \mathrm{T}(t)[\beta, \delta]$ iff $t^{\mathcal{N}} \in$ $V_{\delta}^{+}$and $\models \mathrm{F}(t)[\beta, \delta]$ iff $t^{\mathcal{N}} \notin V_{\beta}^{+}$. The motivation behind this definition is persistence, i.e. that for all $\beta^{\prime}<\beta<\delta<\delta^{\prime}$ and for all sentences $A$, if $\models A[\beta, \delta]$, then $=A\left[\beta^{\prime}, \delta^{\prime}\right]$. In order to have persistence also for sequents we need to define it as follows: $\models \Gamma \Rightarrow \Delta[\beta, \delta]$ iff $\models \bigvee \Delta[\beta, \delta]$ or $\models \neg \wedge \Gamma[\beta, \delta]$. Now the crucial lemma is the following:

Lemma 15. If $\mathrm{SK}^{\infty} \vdash_{\text {lit }}^{\alpha} \Gamma \Rightarrow \Delta$, then $\models \Gamma \Rightarrow \Delta\left[\beta, \beta+2^{\alpha}\right]$ for all $\beta$.

Proof. The proof of the lemma is by induction on $\alpha$. The crucial case is cut where we can use the fact that the cut formula is a literal. The other ingredient for the establishing this fact is persistence. We illustrate the proof structure by considering the case of a cut on $\mathrm{F}$ :

$$
\begin{aligned}
& \mathrm{SK}^{\infty} \vdash_{\text {lit }}^{\alpha^{\prime}} \Gamma \Rightarrow \Delta, \mathrm{F}(t) \text { and } \\
& \mathrm{SK}^{\infty} \vdash_{\text {lit }}^{\alpha^{\prime}} \mathrm{F}(t), \Gamma \Rightarrow \Delta .
\end{aligned}
$$

By IH we get that for all $\beta$,

$\models \Gamma \Rightarrow \Delta, \mathrm{F}(t)\left[\beta, \beta+2^{\alpha^{\prime}}\right]$, which is $=\neg \bigwedge \Gamma\left[\beta, \beta+2^{\alpha^{\prime}}\right]$ or $\models \bigvee \Delta\left[\beta, \beta+2^{\alpha^{\prime}}\right]$ or $\models \mathrm{F}(t)\left[\beta, \beta+2^{\alpha^{\prime}}\right]$.

\footnotetext{
${ }^{10}$ See for Buchholz [2].
} 
And we have for all $\beta$ :

$\models \mathrm{F}(t), \Gamma \Rightarrow \Delta\left[\beta, \beta+2^{\alpha^{\prime}}\right]$, which is $\models \mathrm{T}(t)\left[\beta, \beta+2^{\alpha^{\prime}}\right]$ or $\models \neg \bigwedge \Gamma\left[\beta, \beta+2^{\alpha^{\prime}}\right]$ or $=\bigvee \Delta\left[\beta, \beta+2^{\alpha^{\prime}}\right]$.

We have to show for all $\beta, \models \Gamma \Rightarrow \Delta\left[\beta, \beta+2^{\alpha}\right]$. So let $\beta_{0}$ be arbitrary and $\delta_{0}=\beta_{0}+2^{\alpha^{\prime}}$. Then we have $\left(\models \neg \bigwedge \Gamma\left[\delta_{0}, \delta_{0}+2^{\alpha^{\prime}}\right]\right.$ or $\models \bigvee \Delta\left[\delta_{0}, \delta_{0}+2^{\alpha^{\prime}}\right]$ or $\left.\models \mathrm{F}(t)\left[\delta_{0}, \delta_{0}+2^{\alpha^{\prime}}\right]\right)$ and $\left(\models \mathrm{T}(t)\left[\beta_{0}, \delta_{0}\right]\right.$ or $\models \neg \bigwedge \Gamma\left[\beta_{0}, \delta_{0}\right]$ or $\left.\models \bigvee \Delta\left[\beta_{0}, \delta_{0}\right]\right)$.

If $\models \neg \bigwedge \Gamma\left[\delta_{0}, \delta_{0}+2^{\alpha^{\prime}}\right]$ or $\models \bigvee \Delta\left[\delta_{0}, \delta_{0}+2^{\alpha^{\prime}}\right]$ or $\models \neg \bigwedge \Gamma\left[\beta_{0}, \delta_{0}\right]$ or $\models$ $\bigvee \Delta\left[\beta_{0}, \delta_{0}\right]$ then we get by persistence $\models \Gamma \Rightarrow \Delta\left[\beta_{0}, \beta_{0}+2^{\alpha}\right]$.

The other case is not possible because if $=\mathrm{F}(t)\left[\delta_{0}, \delta_{0}+2^{\alpha^{\prime}}\right]$ and $\models \mathrm{T}(t)\left[\beta_{0}, \delta_{0}\right]$ we would have $t^{\mathcal{N}} \notin V_{\delta_{0}}$ and $t^{\mathcal{N}} \in V_{\delta_{0}}$.

Since $\beta_{0}$ was arbitrary we get for all $\beta, \models \Gamma \Rightarrow \Delta\left[\beta, \beta+2^{\alpha}\right]$.

Now we can argue: If $\mathrm{PKF} \vdash \Rightarrow A$, then $\mathrm{PKF}_{\mathrm{INT}} \vdash \Rightarrow \mathrm{T}(\ulcorner A\urcorner)$ and by partial cut elimination there is a $k$ such that $\mathrm{PKF}_{\mathrm{INT}} \vdash_{l i t}^{k} \Rightarrow \mathrm{T}(\ulcorner A\urcorner)$. By our embedding we get $\mathrm{SK}^{\infty} \vdash_{\text {lit }}^{\omega \cdot k} \Rightarrow \mathrm{T}(\ulcorner A\urcorner)$. With Lemma 15 we can argue that $\models \mathrm{T}(\ulcorner A\urcorner)[1,1+$ $\left.2^{\omega \cdot k}\right]$ which directly establishes $\# A \in I_{\Lambda}^{\omega^{\omega}}$.

The same upper bound can be reached by employing the asymmetric interpretation directly on $\mathrm{PKF}_{\mathrm{INT}}$ replacing Lemma $15 .{ }^{11}$ This second method can also be used to establish directly an upper bound for PKF $F_{\text {INT }}^{t}$ a variation of $\mathrm{PKF}_{\text {INT }}$ with induction restricted to total formulas in the form:

$$
\frac{\Gamma \Rightarrow \mathrm{T}(\ulcorner A(\dot{x})\urcorner), \mathrm{F}(\ulcorner A(\dot{x})\urcorner), \Delta \quad \Gamma, \mathrm{T}(\ulcorner A(\dot{x})\urcorner) \Rightarrow \mathrm{T}(\ulcorner A(x \dot{+} 1)\urcorner), \Delta}{\Gamma, \mathrm{T}(\ulcorner A(\underline{0})\urcorner) \Rightarrow \mathrm{T}(\ulcorner A(t)\urcorner), \Delta} .
$$

Following Cantini's proof in [5] one can show that if $\mathrm{PKF}_{\mathrm{INT}}^{t} \vdash \Rightarrow A$, then $\# A \in$ $I_{\Lambda}^{\omega}$.

\section{Concluding remarks}

Let us summarize what we have done in this paper: We have used an infinitary proof system $\mathrm{SK}^{\infty}$ in order to characterize the minimal fixed-point. The infinitary proof system and the fixed-point construction harmonize neatly, i.e. the height of the derivations correspond directly to the stages of the inductive definition. An upper bound on the height of derivations therefore is also an upper bound on the fixed-point construction. The method applied here seems to be applicable in a wide range of cases. In the paper we focused on the special case of the theory PKF. Whereas the classical theory KF cannot be embedded directly into the infinitary system, because there are KF theorems that are not in the minimal fixed-point, PKF theorems are all elements of the minimal fixed-point. We showed that an embedding can be used to get an upper bound establishing that every theorem of PKF appears in the minimal fixed-point construction at a stage less than $\omega^{\omega}$.

Our work is based on previous work: Infinitary proof systems in the connection with fixed-points were used by Cantini [6], Welch [17] and Meadows [14].

\footnotetext{
${ }^{11}$ Thanks to an anonymous referee for pointing out this variation of the proof as well as the suggestion for a proof of the upper bound for $\mathrm{PKF}_{\mathrm{INT}}^{t}$.
} 
Halbach and Horsten use an interpretation of their PKF in a system they call $\mathrm{KF}_{\text {INT }}$ which is Cantini's system KF. ${ }^{12}$ They use a proof-theoretical analysis of Cantini to show that the arithmetical theorems provable in PKF are those provable in $\mathrm{RT}_{\omega^{\omega}}$. But Cantini has another result which states that if $\mathrm{KF}_{\text {INT }} \vdash \mathrm{T}(\ulcorner A\urcorner)$, then $\# A \in \Gamma^{\omega^{\omega}}$ and if $\mathrm{KF} \vdash \mathrm{T}(\ulcorner A\urcorner)$, then $\# A \in \Gamma^{\epsilon_{0}}$. Combining Halbach and Horsten's interpretation and Cantini's theorem is an alternative to see that every theorem of PKF appears in the minimal fixed-point construction at a stage less than $\omega^{\omega}$.

The theory PKF has its virtues, but it also has its negative aspects. It is much closer to the fixed-point construction in spirit, i.e. as an internal axiomatization of the semantic construction than any of its rival classical theories. Not only the theorems of $\mathrm{PKF}$, i.e. derivable sequents of the form $\Rightarrow A$, harmonize with the fixed-point construction but also all the derivable sequents have a natural reading within the models. If we think of the rules of PKF as resembling our assertoric behaviour, sequents of the form $\Gamma \Rightarrow \Delta$ are to be read as saying that if we are justified in asserting all sentences in $\Gamma$, then we are also justified in asserting the disjunction of all sentences in $\Delta$. The strength and virtue of PKF lies in the derivability of sequents with non-empty antecedent. This is underpinned by the adequacy of PKF.

Let us compare this to Reinhardt's suggestion to focus on the the inner theory of KF also called IKF. ${ }^{13}$ Remember that IKF is the set of sentences such that $\mathrm{KF} \vdash \mathrm{T}(\ulcorner A\urcorner)$. The classical theory $\mathrm{KF}$ plays here a purely instrumental role in the derivation of the significant part, i.e.IKF. This sharply contrasts with natural reading of PKF hinted at in the previous paragraph.

What about the results of the upper bound? If we would only focus on the theorems of PKF, then IKF would seem preferable, because our embedding establishes that already at stage $\omega^{\omega}$ all theorems of PKF are captured, whereas Cantini's result shows that all sentences of IKF appear in the fixed-point construction only before $\epsilon_{0}$. So in a sense this seems to establish that although PKF-theorems and IKF have unintended models, i.e. models which are not fixedpoints, IKF has less and is closer to the minimal fixed-point. However as already mentioned in the case of PKF we have a good motivation to consider all the sequents, whereas in the case of IKF we seem to lack such a motivation.

However it is not surprising that the move away from classical logic also comes with some costs. The discrepancy in the upper bounds also points towards a difference in the proof-theoretic strength of the systems, i.e. the arithmetical theorems. Some authors like Halbach [10] take this to be a serious defect of PKF. A discussion of this point is beyond the scope of this paper. ${ }^{14}$

\footnotetext{
${ }^{12}$ See Cantini [5].

${ }^{13}$ See Reinhardt $[16]$.

${ }^{14}$ For more on this topic see Halbach and Nicolai [12] and Fischer et al. [9].
} 


\section{References}

[1] S. Blamey. Partial logic. In D. Gabbay and F. Guenthner, editors, Handbook of Philosophical Logic, volume 5, pages 261-353. Kluwer, 2 edition, 2002.

[2] W. Buchholz. Skript: Logik I, 2001.

[3] J. P. Burgess. The truth is never simple. The Journal of Symbolic Logic, 51:663-681, 1986.

[4] S. R. Buss, editor. Handbook of Proof Theory. Elsevier Science Publisher, 1998.

[5] A. Cantini. Notes on formal theories of truth. Zeitschrift für mathematische Logik und Grundlagen der Mathematik, 35:97-130, 1989.

[6] A. Cantini. A theory of formal truth arithmetically equivalent to $\mathrm{ID}_{1}$. The Journal of Symbolic Logic, 55:244-259, 1990.

[7] H. Field. Saving Truth from Paradox. Oxford University Press, 2008.

[8] M. Fischer, V. Halbach, J. Kriener, and J. Stern. Axiomatizing semantic theories of truth? Review of Symbolic Logic, 8:257-278, 2015.

[9] M. Fischer, L. Horsten, and C. Nicolai. Iterated reflection over full disquotational truth. to appear in Journal of Logic and Computation, 2017.

[10] V. Halbach. Axiomatic Theories of Truth. Cambridge University Press, Cambridge, UK, revised edition, 2014.

[11] V. Halbach and L. Horsten. Axiomatizing Kripke's theory of truth. The Journal of Symbolic Logic, 71:677-712, 2006.

[12] V. Halbach and C. Nicolai. On the costs of nonclassical logic. Journal of Philosophical Logic, 2017. Online first https://link.springer.com/article/10.1007/s10992-017-9424- 3.

[13] S. Kripke. Outline of a theory of truth. The Journal of Philosophy, 72:690$716,1975$.

[14] T. Meadows. Infinitary tableau for semantic truth. Review of Symbolic Logic, 2015.

[15] G. Priest. In Contradiction. Oxford University Press, 2 edition, 2006.

[16] W. N. Reinhardt. Some remarks on extending and interpreting theories with a partial predicate for truth. The Journal of Philosophical Logic, 15:219-251, 1986 .

[17] P. Welch. Games for truth. Bulletin of Symbolic Logic, 15(4), 2009. 